\title{
Designing for Gold Plating
}

\author{
INFLUENGE ON COST AND RELIABILITY
}

Harold Silman

Industrial Consultant, London

Good design plays an important part in ensuring that gold-plated products or components are produced efficiently and that they perform satisfactorily in service. The various design parameters that influence the electrodeposition of gold and the quality of the plated finish are discussed in detail and examples are given of the principal difficulties encountered by electroplaters and of how they can be eliminated, or at least appreciably reduced, by attention to design.

Design plays an important part in electroplating, not only in keeping down costs but in ensuring that the product will have a good appearance and perform satisfactorily in service. This is especially true in the case of gold plating, where the cost of the metal makes it essential that the deposit should be as uniform as possible so as to meet the minimum thickness specifications increasingly demanded without unnecessarily exceeding them at any point. It is desirable that the designer should be conversant with the basic principles of the processes to be employed, and their limitations, as well as with the conditions of service to which the plated articles are to be subjected, to ensure that plating can be carried out efficiently and economically. He should co-operate with the electroplater at the design stage to avoid difficulties which may subsequently prove costly to overcome, result in high reject rates, and yield an unsatisfactory product.

Finishing processes are not cheap to carry out, especially in the case of gold plating to specification. Good design enables good finishes to be applied, and small changes can often be made which can help enormously in simplifying production and in reducing costs. On the other hand the designer should not allow himself to be submerged by fear of problems which his designs may create in production; much progress in finishing techniques has resulted from the challenge presented by "impossible" designs. A good example of a complicated design, dictated by service requirements, but one which successfully challenged the ingenuity of the plater, is shown in Figure 1.

Gold plating is becoming increasingly important for industrial applications, and a wide variety of processes is available, each having its advantages for particular purposes. It is not proposed to discuss them in detail, since the general considerations with respect to design apply to a greater or lesser extent to all of them.

\section{Mechanical Finishing}

Most articles have to be given some kind of initial mechanical finish, such as grinding or polishing, before they are plated. This is generally carried out by means of abrasives on suitable belts or wheels, either by hand or on automatic or semi-automatic machines. For special purposes, a variety of blasting procedures is available while, for the finishing of articles in bulk, barrel polishing or the newer vibratory finishing methods may be employed. In this way all kinds of surface imperfections are removed and the articles made ready for the plating process.

The following are some of the more important aspects to consider in mechanical finishing:

(a) Abrupt changes in shape, sharp edges (which can damage wheels and belts), and blind holes or joint crevices (which can retain polishing compound) are to be avoided.

(b) Large flat areas should be broken up in some way so as to make them less liable to scratches and minor damage. It is also difficult to produce uniformly flat reflecting surfaces by polishing, while they are also susceptible to subsequent damage.

(c) Projections and protuberances should be avoided since they are difficult to polish and may damage polishing buffs.

(d) Deep recesses and re-entrant areas should be avoided as far as possible, and all surfaces to be polished should be readily accessible to polishing wheels of normal diameter. Fine surface patterns are also undesirable, as they may be lost or blurred in polishing. 
Fig. 1 While good design enables electroplating to be carried out efficiently and economically, complicated designs dictated by service requirements can sometimes lead to progress in plating techniques. This U.H.F. resonant cavity from an airborne transponder, designed and made by Cossor Electro. nics, is cast in a siliconaluminium alloy and machined to a high finish on the significant surfaces. It is then electroplated with nickel, silver and finally gold. In order to achieve uniformity in deposit thickness in quantity production the electroplating, carried out by Process Services, involved the use of three internal anodes and one external anode, all operating at different currents and requiring complex circuitry to achieve the required result



(e) Articles intended for bulk polishing should be sufficiently strong to withstand the tumbling action, and should not stick together or interlock. A dimple or a raised edge is useful on flat surfaces to prevent them sticking together.

\section{Design for Racking}

All articles, except those to be plated in barrels, have to be racked for transfer through the degreasing, cleaning, plating, rinsing and drying cycles, and must therefore be suspended from suitable racks. First of all it is necessary to establish the "significant surface", that is the part of the component which is essential to its appearance or serviceability. It must then be designed in such a way that it can be held securely on the plating rack with good electrical contact, and without preventing the plating of any part of the significant surface. Sometimes the designer may have to incorporate lugs to enable proper contact to be made, these being removed after finishing. This, however, should rarely be needed.

Another important point is that provision must be made for drainage from the racked part; hence it is necessary that the designer should have an indication as to how the articles are to be suspended from the racks so that provision can be made for drainage holes if possible. Trapping of solution is to be avoided, since it causes contamination by carry-over, as well as by changing the composition of solutions and causing a wastage of chemicals. It can also lead to corrosion by the entrapment of residues and salts. Air entrapment must also be prevented, since this can cause unplated areas to occur, while gas can accumulate in such areas during cleaning and plating.

In the case of articles to be barrel plated, there are special requirements in addition to those stated above for plating on racks. The articles should be suffi-

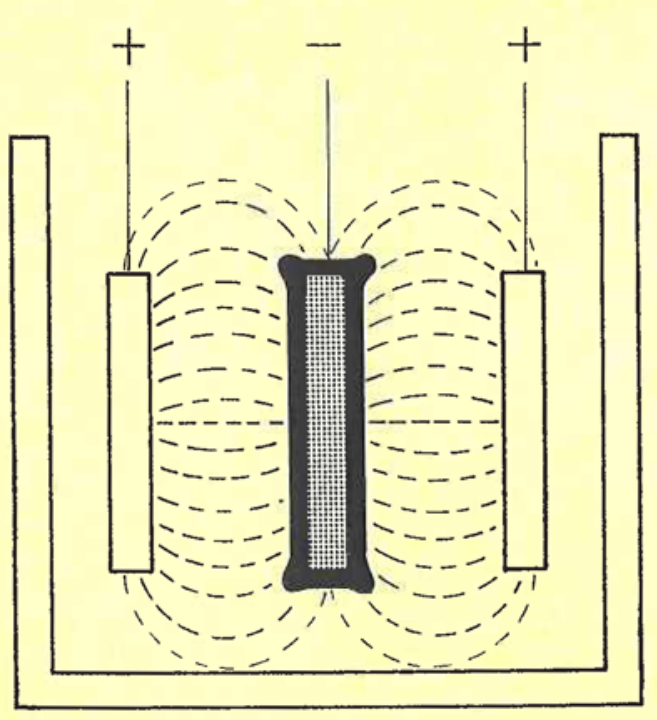

Fig. 2 A cathode consisting of a flat plate, positioned parallel to an anode in an electroplating solution, should theoretically receive a deposit of uniform overall thickness. In practice, however, there will be a concentration of current flow at the edges, giving a greater thickness of deposit in these areas 


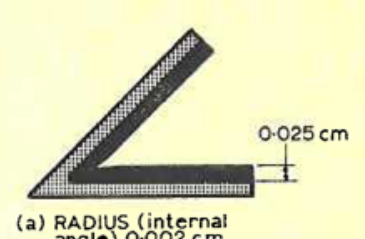

(a) RADIUS (internal
angle) $0.002 \mathrm{~cm}$ (1)

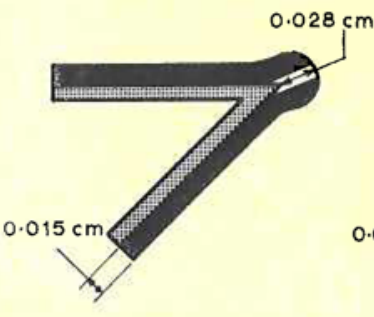

(c) RADIUS (external
angle) $0.08 \mathrm{~cm}$

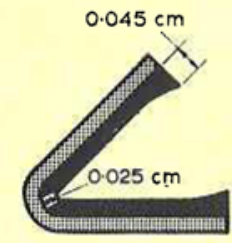
(b) RADIUS (internal
Fig. 3 The effect of angle of curvature on the distribution of thickness of deposit on the internal and external angles of a cathode bent at $45^{\circ}$, showing the improvement in distribution with increasing radius

ciently robust not to be damaged during the rotation of the barrels, while their design should be such as to prevent them from nesting or sticking together. Where the barrel plating of mixed loads cannot be avoided the articles should not be of widely differing dimensions, since the larger parts will tend to receive a higher proportion of the deposited metal while the build-up on high density areas will also be increased.

\section{Metal Distribution}

The performance of a coating is highly dependent on the thickness of metal applied to the significant surface, which is determined by certain basic laws. In general, smooth-flowing shapes are conducive to obtaining good metal distribution, while any protuberances such as corners and sharp edges will receive disproportionately thick deposits. On the other hand, concave areas, grooves and deep recesses will tend to be underplated. This can be compensated for by increasing the plating time, but has the disadvantage that unnecessary thicknesses of gold will be deposited on other areas where it may not be required, while plating costs are correspondingly increased by the reduced production rate. If a flat plate is made the cathode parallel to an anode of large surface area in a plating solution it should theoretically receive a deposit of uniform overall thickness. In practice, however, there will be a concentration of the lines of current at the peripheral zones, leading to a greater thickness of deposit at these locations. Figure 2 shows the distribution of current and deposit on a rectangular section cathode. The distribution of metal can also be affected by disturbing the film of solution at the cathode surface; such hydrodynamic streaming has an influence on the thickness of the cathode film and hence on the equilibrium current density and the deposit thickness. This effect has been studied by Keulegan (1).

The investigation of three-dimensional electrode systems has been carried out with the aid of analogue methods by Rousselot (2) who studied the primary current distribution on the side walls of a V-shaped notch. Agitation of the solution affects the cathode film thickness, and hence the metal distribution. The possibilities of computers for the investigation of current distribution are a comparatively recent development; the method offers valuable assistance in the resolution of the very complex phenomena involved in the calculation of current distribution.

Figure 3 shows how the deposit thickness distribution varies within a $45^{\circ}$ angle of different curvatures. The radius of curvature in (a) is 0.002 $\mathrm{cm}$ and in (b) it is $0.016 \mathrm{~cm}$. The corresponding thickness distribution on the external surfaces of a
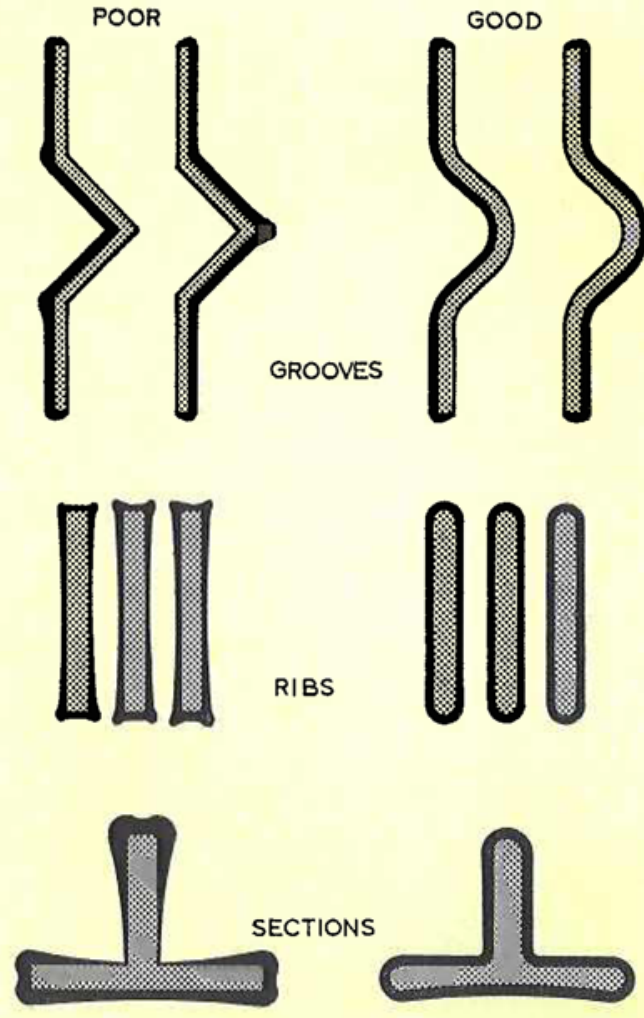

Fig. 4 Some typically poor design features that can lead to uneven distribution of thickness of an electrodeposit, and the simple means that can be adopted to ensure more even distribution 
Fig. 5 Small components are generally gold plated in a rotating perforated barrel made of plastic material and immersed in the plating tank. The barrel has flexible leads to enable the current to pass to the individual parts, which are in contact with each other. These should therefore be sufficiently robust not to be damaged during barrelling, while their design should be such as to prevent them from nesting or sticking together

Photograph by courtesy of Johnson Matthey \& Co Limited

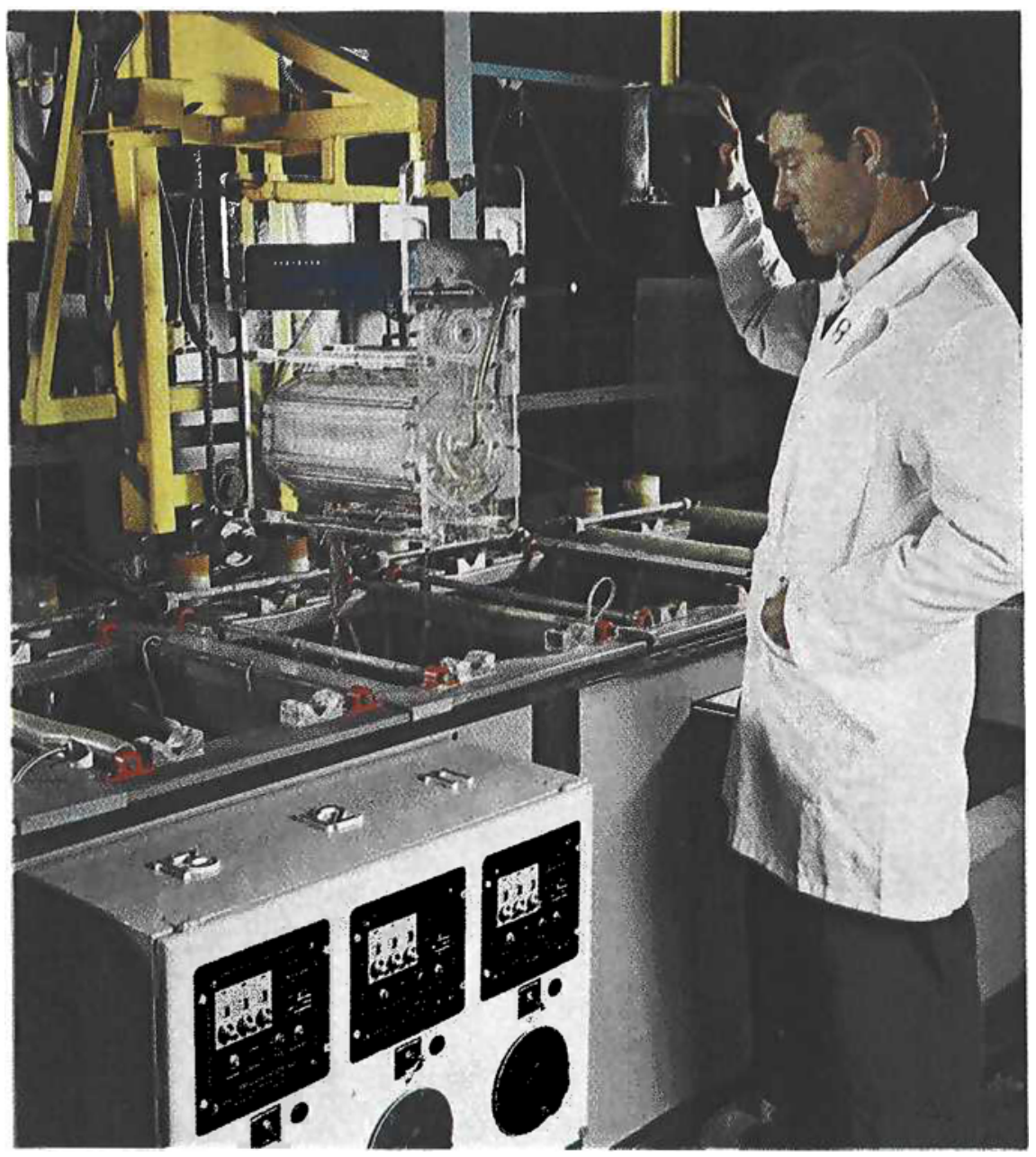

$45^{\circ}$ angle can be seen in (c) and (d), where the radii are 0.08 and $0.22 \mathrm{~cm}$ respectively. The improvement in the distribution resulting from increasing the radii is readily seen.

Some typical design features which can lead to poor metal distribution, and means which can be adopted to effect an improvement, are shown in Figure 4. Although this work was carried out on copper plating solutions, the same considerations apply in principle to gold plating processes of every kind.

Although some improvements can be effected in metal distribution by modifications to the plating bath composition, design plays a very important part in obtaining a good deposit; the area of thinnest coverage is most liable to the affects of abrasion, wear and corrosion, and for this reason all plating specifications demand minimum deposit thicknesses.

In general convex surfaces are the ideal shapes on which to apply electrodeposits, especially when they have rounded edges. Flat surfaces are not easy to polish, while surface defects are readily visible on them; even a slight curvature of the surface will reduce the effects of any unevenness which may appear during polishing.
All edges should be rounded; sharp edges are undesirable, since the coating thickness is reduced in the central areas, while an increased plating time becomes necessary for obtaining the minimum coating thickness. Sharp-edged recesses likewise increase both the plating time and the costs of producing the minimum coating thickness demanded. All types of projections have an unfavourable effect on the uniformity of the coating thickness. Blind holes cannot generally be plated with the specified minimum thickness of deposit since a disproportionately high deposit would have to be applied on the rest of the surface in order to obtain the required thickness of deposit in the recess; V-shaped grooves and sharp recesses in large areas should also be avoided as far as possible.

\section{Polarisation Effects}

Polarisation has an important effect on the current distribution. A distinction can be made between macro- and micro-throwing power. Thus cyanide and sulphite gold solutions have good macrothrowing power, and uniform distribution of the deposit is produced even on recessed areas. How- 


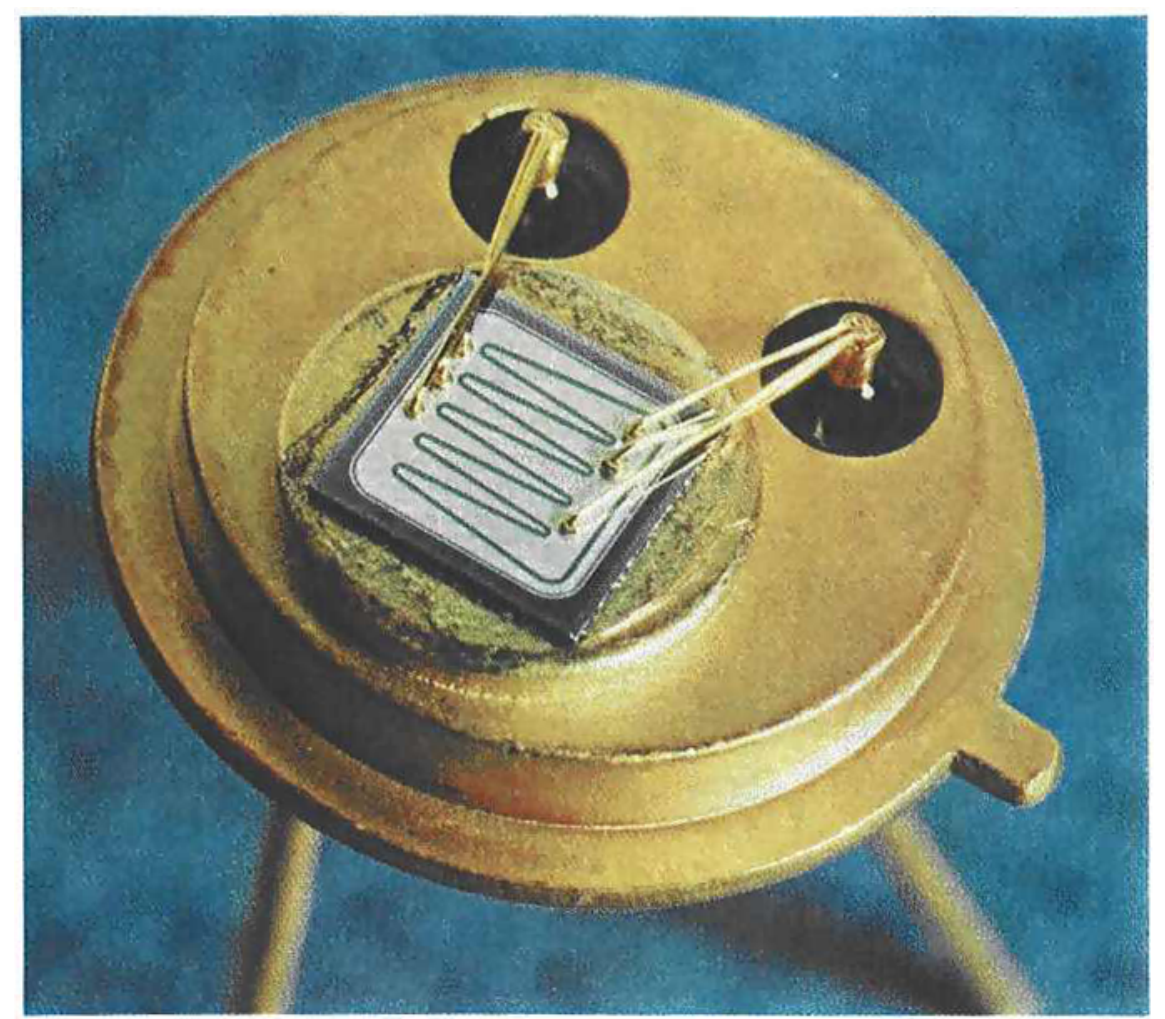

Fig. 6 The design of transistor headers makes them more suitable for plating on racks, but generally the pressures on production result in them being barrel plated. The leads then tend to interfere with tumbling, so that there may be a wide spread in the thickness of gold deposited. This effect is aggravated by the fact that only one of the leads is in electrical contact with the header

ever, the micro-throwing power of the solution is low, so that minor imperfections and scratches are not covered by the deposit. In addition to the primary current distribution, secondary lines of current distribution occur as a consequence of the specific chemical and electrochemical properties of the electrolyte in so far as their tendency towards polarisation is concerned. Geometrical effects of the anode/ cathode orientation and their relative dimensions also play a part in this. Finally, there are tertiary lines of current distribution, which are a function of the cathode efficiency of the bath.

\section{Some Basic Rules}

The following are some basic rules for the design of articles for gold plating in order to achieve maximum economy and reliability:

(a) Deep recesses and abrupt re-entrant areas should be avoided as far as possible, particularly where the deposit has to resist wear or corrosion. Recesses and their edges should be rounded off to a radius of at least $0.2 \mathrm{~cm}$; the minimum radius at the edge and base of an indentation should be one-quarter of the depth (obtuse angles present less problems than acute angles). Where minimum deposit thicknesses are specified, they are usually not applied to areas which cannot be touched by a ball of $20 \mathrm{~mm}$ diameter, this being the standard adopted in ISO specifications. (b) Tubular articles should either be completely sealed or should have provision for complete drainage to prevent carry-over of process solutions during plating.

(c) Blind holes, cavities, seams and rolled edges which can trap process solutions should be avoided. The space between adjacent holes should preferably be at least twice their diameters; holes and slots should be free from sharp edges. Articles should be assembled after plating, particularly if they are made of dissimilar metals.

(d) The design should take into account the need for providing a suspension point giving good electrical contact on the article to be plated when suspended by its own weight. This may be a hole, a thread or a lug located in a position where the surface appearance is relatively unimportant.

(e) Convex surfaces are more readily plated than flat or concave ones. A crown of at least $0.040 \mathrm{~cm}$ is recommended. If a concave surface is essential, its depth should not exceed half its width.

(f) Protuberances, sharp corners, edges, fins and ribs, draw current preferentially, and will therefore tend to build up with metal during plating. They should therefore be rounded with a radius of at least $0.1 \mathrm{~cm}$, while edges of holes can be countersunk or chamfered. 
(g) The electroplating of screw threads presents special problems. The distribution tends to be non-uniform, which can result in assembly difficulties.

The table shows the minimum radii which have been recommended by Safranek and Underwood for angles in recesses in order to obtain satisfactory electroplating (3).

\section{Minimum Radii at Angles in Recesses}

Depth of Recess
$(\mathrm{mm})$
1.6
3.2
6.4
9.6
12.8

Minimum Radius of the Angle between Opposite Surfaces ( $\mathrm{mm}$ )

0.4

0.8

1.6

2.4

In accordance with ISO specifications, the minimum coating thickness specifications apply, however, only to surfaces which can be touched by a ball of $20 \mathrm{~mm}$ diameter.

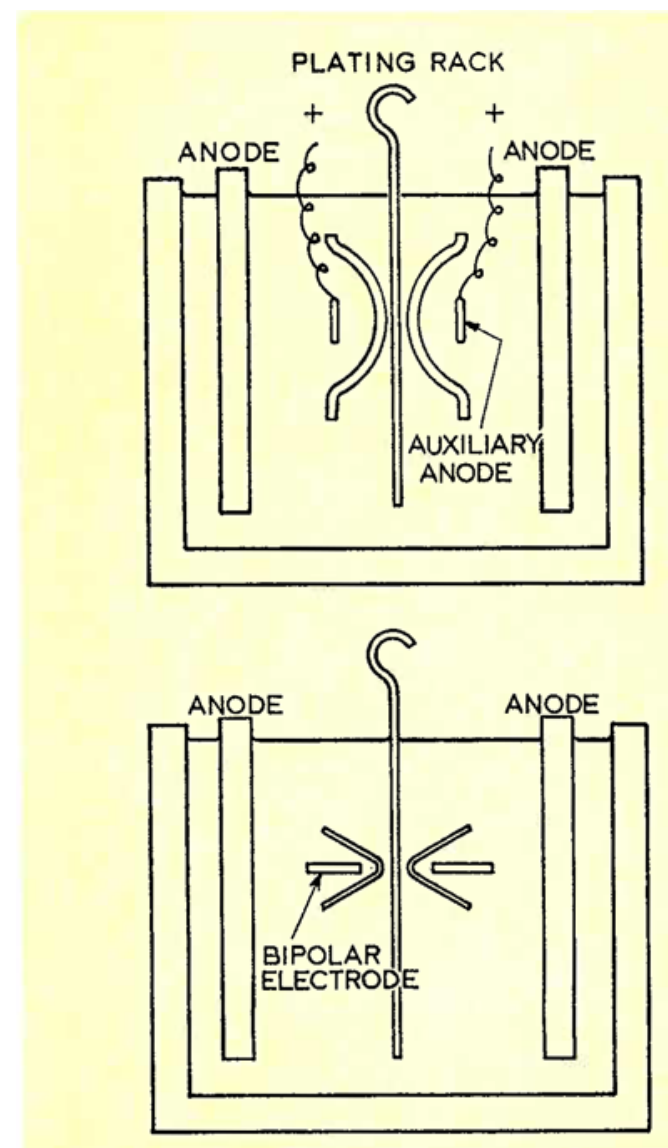

Fig. 7 In the plating of recessed or concave surfaces it may be necessary to use either auxiliary anodes or, in some cases, bi-polar electrodes of an inert metal, to assist in leading the current to where deposition is needed
The design of die-cast components for good plating requires that careful attention should be given to the essential features of the die itself. The parting of the die should not occur on flat surfaces, but at edges or raised areas. The same applies to gates and air vents, as well as to blind holes. It should be possible to remove casting sprues and bores by grinding without penetrating the skin of the casting. Recessed flat areas should be avoided, and a uniform wall thickness of 0.8 to $3 \mathrm{~mm}$ should be aimed for. There is nowadays a trend towards castings with thinner walls, with a view to reducing weight and cost. Sharp cross-sectional changes in the casting are also undesirable, since they interfere with metal flow; this factor is improved by the rounding of corners and edges.

It is frequently necessary to cast inserts into zinc die-cast articles either to strengthen them or to provide attachments such as bushes or screw threads. Projecting threads tend to build up in metal when plated, and this must be allowed for in specifying the tolerances of the plated article. The casting-in of metals such as steel or brass can also result in cleaning problems since the cleaning procedures for these metals may differ from those for zinc die-castings. This difficulty can be obviated by zinc plating inserts before casting-in so that the entire article to be plated can in effect be treated as made wholly of zinc.

Metal also tends to build up around the edges of holes in die-castings. This effect can be reduced by making the bores slightly tapering. In general, it can be said that designs that are suitable for die-casting are also favourable to good electroplating.

\section{Auxiliary Anodes}

Where recessed or concave surfaces have to be plated it may be necessary to employ auxiliary anodes. Such anodes present problems in attachment, and they slow down production, so that their use is to be avoided if at all possible. Gold rods or wires are generally employed, with copper or nickel for appropriate undercoat deposits where these are required.

Bipolar electrodes are also useful in some cases. These are simply bars or strip of an inert metal which serve the purpose of leading the current to where it is wanted. Bipolar electrodes are not connected electrically to either the anode or the cathode. The use of both auxiliary and bipolar electrodes is illustrated diagrammatically in Figure 7.

\section{Plating Articles in Bulk}

Articles for barrel plating should be sufficiently strong not to be damaged by impact during rotation of the barrel. Flat parts are liable to nest together, but this tendency can be reduced by the introduction of design features such as ridges or small projecting 


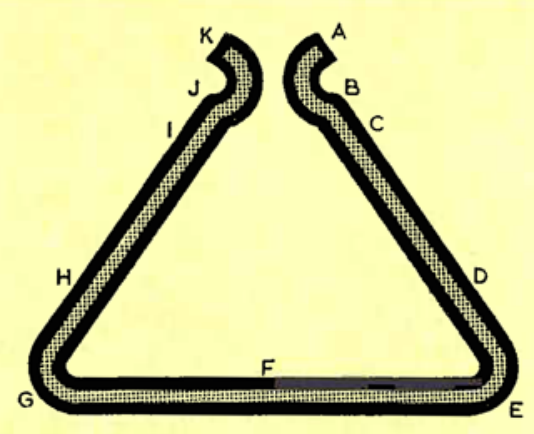

Fig. 8 The variation in thickness of gold deposit on a barrel plated phosphor bronze spring contact

$\begin{array}{lllllllllllll}\text { Point } & \text { A } & \text { B } & \text { C } & \text { D } & \text { E } & \text { F } & \text { G } & \text { H } & \text { I } & \text { J } & \text { K } \\ \begin{array}{l}\text { Thickness } \\ \text { (internal) } \mu \mathrm{m}\end{array} & 10 & 8 & 8 & 10 & 7 & 12 & 7 & 10 & 8 & 11 & 11 \\ \begin{array}{l}\text { Thickness } \\ \text { (external) } \mu \mathrm{m}\end{array} & 10 & 9 & 7 & 8 & 8 & 9 & 8 & 8 & 7 & 9 & 11\end{array}$

areas. Mixed loads should also be avoided, as different articles will become plated with different thicknesses of deposit; larger articles, in particular, generally receive greater thicknesses.

Deposit thickness distribution in barrel plating is a special case. Generally the coating thickness variation tends to increase as the deposit thickness increases. Figure 8 shows the metal distribution on a typical barrel plated spring contact. For a proper assessment of the mean thickness of the deposit on articles in a barrel load, the testing of at least 15 samples may be necessary, while 20 to 25 articles may well be required for the calculation of the standard deviation.

\section{Gold Reflectors for Lasers}

The many applications of high-power-density lasers have introduced more severe requirements for optical reflectors. In particular there is now a need for reflective surfaces consisting of high purity metals free from even minute defects such as inclusions or voids which might lead to damage. The preparation of such high quality optical surfaces by a newly developed type of precision machining of an electrodeposited layer of gold is being actively pursued at the Oak Ridge Plant in Tennessee, and a progress report from there by F. B. Waldrop, M. J. Bezik, W. E. Tewes and R. C. Waldrop gives some details of the procedures adopted (Applied Optics, 1975, 14, (8), 1783-1787).

The high quality machined surface is obtained by using special machine tools and tool bits that are ground from single crystal diamond, the process being referred to as diamond turning. As yet only copper, silver, gold and aluminium are suitable for machining by this method, but a more refractory metal such as beryllium (to reduce weight) or molybdenum (to achieve a low coefficient of expansion) is desirable for the substrate. These metals are of course generally characterised by a tenacious oxide film that militates against adhesion of
An important factor in thickness distribution is a smooth rolling movement during the plating operation. Complicated shapes cause a shielding effect and large thickness variations, especially when the plating times are short (4). The form of the internal surface of the barrel, the rate of rotation, and the depth of the load are also significant.

\section{Benefits from Good Design}

The question of design for gold plating industrial products has been somewhat neglected when compared with the amount of attention which has been given to designing articles intended for plating with base metals. Even in the latter case, however, much more could be done. Yet the benefits which can accrue from proper attention to design-metal savings, the reduction of rejects, and economies of production costs-generally are of outstanding importance in the case of gold plating.

The adoption of some of the measures which have been outlined will certainly involve some trouble, and possibly a little expense, but these will be vastly exceeded by the resulting gains.

\section{References}

1 G. H. Keulegan, "Hydrodynamics of Cathode Films" F. Res. Nat. Bur. Stand., 1951, 47, (3), 156-169

2 R. H. Rousselot, "Current Distribution over ThreeDimensional Electrodes", Trans. Inst. Metal Finish., 1964, 42, 100-106

3 W. H. Safranek and A. A. Underwood, "Influence of Design on Electroplating of Zinc Diecastings", American Zinc Institute, New York, 2nd Edn., 1966

4 A. W. Wallbank and D. N. Layton, "The Plating of Screw Threads", Trans. Inst. Metal Finish., 1955, 32, 308-335

an electrodeposit. This disadvantage has been overcome by a preliminary vapour deposition process-ion plating-in which an initial very thin but adherent coating of aluminium is produced, upon which a conventional electrodeposit can be applied. This process is carried out in an argon atmosphere under a pressure of a few microns. The surface to be treated is first cleaned by sputtering with argon ions to remove any oxide film. Aluminium ions are then accelerated towards the substrate by the high voltage with such velocity that they penetrate the surface, so ensuring excellent adhesion to the most refractory of metals.

Electrodeposition on to this preliminary coating can then be carried out and good adhesion can be achieved, using standard processes. The most suitable electrolyte has been found to be the citrate-based acid cyanide bath, and deposits from this electrolyte showed no voids under the microscope, a purity of 99.98 per cent, and a density equivalent to that of solid gold.

These gold surfaces, diamond turned to a mirror finish, gave reflectivities of approximately 99.3 per cent at $10.6 \mu \mathrm{m}$ in the as-machined condition, an outstanding quality in terms of reflectivities. 